\title{
Research on Application of Electricity Emergency Communication Based on Multi-situation Awareness
}

\author{
Junxiao Shi, Chenfan Weng, Hui Wu \\ Determent Operation and Maintenance, Information and communication Branch, State Grid Zhejiang Electric Power Corporation, Hangzhou, \\ China
}

\author{
Email address: \\ shi_junxiao@zj.sgcc.com.cn (Junxiao Shi),wengchenfan@ncu.edu.cn (Chenfan Weng),wu_hui@zj.sgcc.com.cn (Hui Wu)
}

\section{To cite this article:}

Junxiao Shi, Chenfan Weng, Hui Wu. Research on Application of Electricity Emergency Communication Based on Multi-situation Awareness. American Journal of Science, Engineering and Technology. Special Issue: Machine Learning in the Internet of Things. Vol. 5, No. 2, 2020, pp. 61-68. doi: 10.11648/j.ajset.20200502.13

Received: February 7, 2020; Accepted: February 24, 2020; Published: April 14, 2020

\begin{abstract}
In order to meet the demand of contemporary emergency communications, the investigations on communication methods, basic network survivability, coverage ability and transmission rate are proposed. The digital microwave, mesh networks, small base stations and portable integrated base station technologies are studied, then multi-situation awareness networking architecture is presented, and the key modules which involved in the solution is discussed. According to the architecture, concept of typical power emergency scene based on natural disasters and transmission line repairs are designed. This paper discusses the practical application of an emergency communication network that can be quickly set up, integrated earth observation system with multi-level command, which meets the higher requirements of future electricity emergency communication guarantee. It is foreseeable that in the future, power emergency communications will present a trend of polymorphism. The networking scheme proposed in this paper has greatly improved the efficiency of establishing the on-site emergency system. In extreme disaster situations, various sensing technologies will take advantage of the unique advantages of their respective technologies, so that multi-level command can be achieved at the company's provincial, city, and even county levels. At the same time, while ensuring the key target area, it can achieve a wide coverage which has certain promotion value.
\end{abstract}

Keywords: Electricity Emergency Communication, Coverage Area, Transmission Rate, Digital Microwave, Small Cell, Mesh Network, Multi-situation Awareness

\section{Introduction}

Emergency communication generally refers to the comprehensive use of various communication resources to ensure rescue, emergency rescue and necessary communication when the demand for artificial or natural emergencies suddenly increases. It is a temporary special communication mechanisms provided in response to natural or man-made emergencies [1]. It has the characteristics of emergency communication capacity, geographical area, coverage network uncertainty, and information diversity. How to quickly set up an emergency communication network with a throughput that meets the requirements of large bandwidth, short network construction time, wide coverage area, and diverse collection terminals under the condition of power failure and no operator network, in order to respond to sudden natural disasters and emergency rescue, has become a problem that needs to be addressed in emergency support.

Electricity emergency communication belongs to the category of regional space emergency communication of electricity infrastructure. The regional space is a floating platform as a carrier, which can recover the information communication destroyed on the ground and solve the remote command and scheduling problem based on video and voice [2]. At present, electricity emergency communication mainly adopts a combination of ground wire communication and satellite communication. The main business is voice telephony, cluster intercom, video and audio consultation, live video image return and office production data, etc. The data types of the business are summarized as voice, video and data business.

The main problems faced by electricity emergency communications are single communication method and system function currently. Mainly relying on basic 
communication facilities, low self-organization and survivability of the network, and poor service type, capacity and quality support capabilities also become the important problems for electricity emergency communications. Without a unified and comprehensive coverage communication system, it is difficult to quickly rebuild the network when the wired communication network is damaged [3]; Although the satellite system is relatively robust and covers a wide area, it is difficult to deploy for all demand units in need because of the limited transmission capabilities, high deployment and usage costs, and difficult technical support. There is clustered communication network within a certain range, however their coverage and communication capacity are small, which are usually limited to scheduling and command and cannot meet the requirements of a large-scale emergency communication network [4].

This paper discusses the changes in electricity communication support requirements, and analyzes the evolution of key technologies involved in existing wireless communications. Based on the mainstream wireless communication frequency bands in electricity, and combined microwave communications, satellite communications, cellular network communications, and mesh technologies, a typical emergency communication guarantee scheme via cross-band large-capacity microwave transmission is proposed. And according to typical scenarios such as natural disasters and transmission line repairs, the network architecture is designed to realize the integrated emergency communication network of heaven_and_earth to meet the higher requirements of future electricity emergency communication guarantee.

\section{Key Technology Evolution}

The "Thirteenth Five-Year Plan" period is the key point for China to further promote the "five modernizations". Production is concentrated. Once an emergency occurs, it will easily makes a chain effect, which will have a huge impact. In extreme weather scenarios, emergency response areas face the problems of no electricity, no public network and no roads. The affected areas changing frequently is also a problem for typhoon disasters. Moreover, the electricity emergency communication support is developing in the direction of the informatization and the longitudinal connection in provinces-counties, which requires high realtime requirement and rapid on-site deployment [5]. At the same time, two major changes occurred in the emergency command mode: hierarchical command changed to flat command, and language command changed to video command. In terms of business requirements, the emergency communication network needs to carry some business such as fixed telephones, digital voice, video conferencing, surveillance images and production monitoring. Under the premise of improving the security of the telecommunications network as much as possible, the coverage of the entire disaster area network is increased. It guarantees remote communication across regions, and supports the contact and data transmission of on-site repair personnel in the absence of electricity and network at the same time.

In summary, the evolution of the demand for emergency communications puts forward higher requirements on the four aspects of future emergency communications support system: diversification of communication methods, basic network survivability, coverage, and transmission rate. The four technologies for the aspects mentioned above will be discussed in this section.

\subsection{Cross-band Aggregation Digital Microwave Technology}

Microwave communication has certain advantages in the range of line-of-sight transmission. The traditional digital microwave system has such defects as insufficient capacity, low reliability and blocked signal transmission channel while the digital microwave technology of cross-band aggregation has many advantages such as large transmission capacity, flexible deployment mode, fast construction speed and less environmental impact. The microwave technology discussed in this paper is used to set up transmission channels between fourth generation mobile communication (4G) base stations, which can save fiber resources. Its jump is based on hierarchical quality of service (HQoS) technology. HQoS is introduced to the user access side and the network bearer side. When multiple emergency business Virtual Private Networks (VPNs) share the same link and bandwidth, multilevel bandwidth and priority scheduling can be used on the user side and network-side outbound, users and business are subdivided to allocate queue resources at different levels, and each level of bandwidth and priority forwarding behavior can be guaranteed, so that the different business can be distinguished from bandwidth and QoS guarantees [6]. However, the security of this technology needs to be improved.

With the development of mobile broadband and fixed broadband networks, the backhaul capacity of base stations has doubled, and the challenges facing microwave in the $5 \mathrm{G}$ era are increasing. Conventional microwaves support transmission distances up to 100KM. They have high reliability and can match the transmission requirements of macro stations, but the spectrum resources are becoming tighter and the transmission bandwidth is low $(<1 \mathrm{Gbps})$, and it is becoming increasingly difficult to expand capacity. Eband (E-band, 71-76 / 81-86GHz) microwaves can support up to $20 \mathrm{Gbps}$ transmission capacity through cross-polarized interference cancellation technology to meet 4G / 5G capacity requirements, but the transmission distance is generally less than $5 \mathrm{KM}$ and cannot meet long distances demand for macro station return.

Super Dual Band [7] is a new type of cross-band aggregation technology. By combining technologies such as physical link aggregation, amplitude modulation AM, and QoS guarantee, combined the conventional frequency band microwave link (6-42GHz) and E-band (71 -76/ 81-86GHz) and applied it to achieve large bandwidth, long distance and highly reliable microwave transmission. The conventional frequency band microwave link and the E-Band microwave 
link form a mutual protection mechanism to ensure that the conventional frequency band microwave link can remain unblocked and high priority service transmission can be guaranteed in long-distance transmission, even if the weather and other factors affect the link.

As shown in Figure 1, a maximum air interface capacity of
20Gbit / s can be provided by the current Super Dual Band. Through the physical link aggregation between conventional frequency band links and E-band links, the bandwidth utilization rate can be improved, and cross-band AM can be achieved, and core business can be guaranteed through QoS.

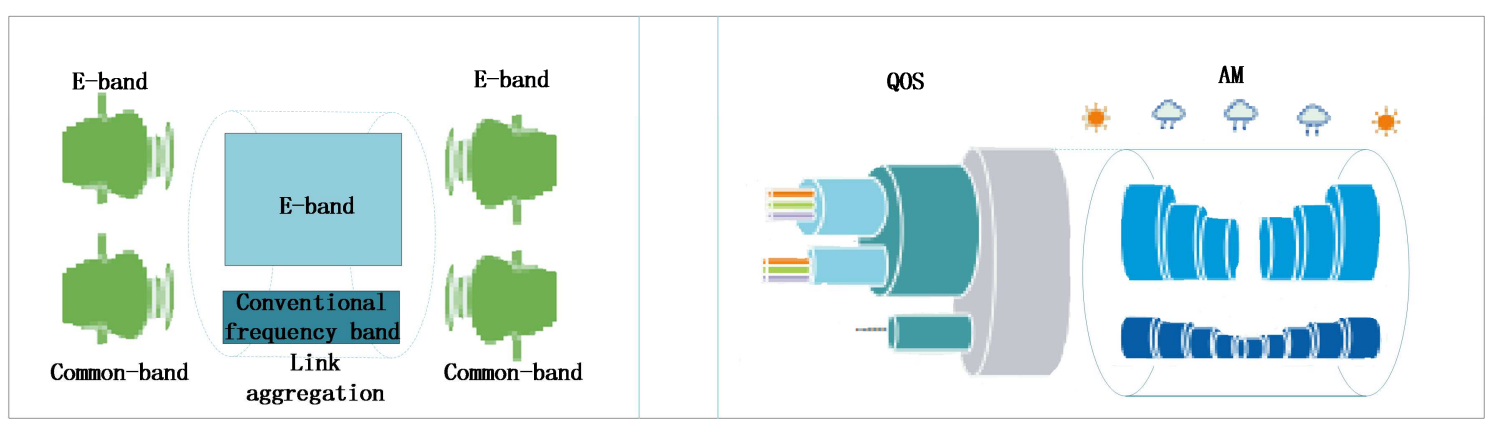

Figure 1. Schematic diagram of microwave technology.

\subsection{The Radio Frequency Mesh (RF-Mesh) Technology}

The RF-Mesh technology uses the characteristics of the non-center multi-hop ad hoc network to achieve the rapid establishment of multi-node IP interconnection and link backhaul in non-signal areas. It is the product of the integrated development of mobile communications and computer networks [8], of which ad hoc networks, Wireless Sensor Networks (WSN) and Wireless Mesh Networks (WMNs) all belong to the technical category of wireless ad hoc networks [9], where Mesh networks are a new type of wireless local area network. Different from the traditional wireless local area network, the wireless access points (APs) in a wireless mesh network can be interconnected by wireless connections, and bidirectional multi-hop wireless links can be established between APs [10]. It supports a point-tomultipoint mesh structure with intelligent advantages such as ad hoc networking, self-repair, multi-hop cascading, node self-management as well as mobile bandwidth and wireless positioning [11].
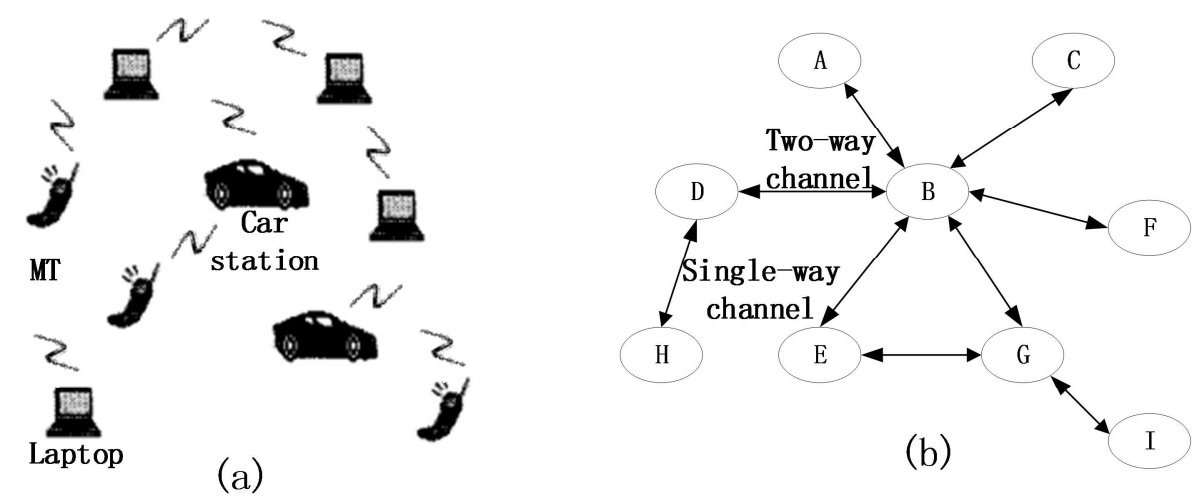

Figure 2. Self-organizing network topology.

It can be seen from Figure 2 that the wireless ad hoc network is a multi-hop temporary autonomous system composed of a group of mobile terminals with wireless transceivers. The mobile terminal has both the functions of a router and a host and Network topology can be formed by wireless connections. This network can work independently and can also be connected to the Internet or cellular wireless networks [12]. In addition, the RF-Mesh wireless ad hoc network technology can be used as an extended network of Long Term Evolution (LTE). For blind spots of LTE signals such as underground or in tall buildings, the RF-Mesh wireless ad hoc network technology is used to extend blindness to achieve video backhaul and command scheduling of complex scenes such as inside buildings, underground and no-signal areas.

\subsection{LTE Wireless Small Cell}

In the cellular network, electricity emergency communication is mainly carried out on the second generation of mobile communication applications. Based on air interface technologies such as GPRS / GSM, it is carried on macro base stations of communication towers [13]. The small base station has small transmit power and radiation range, and flexible product deployment. And the average power consumption of the small base station is much lower 
than that of the traditional base stations. As an important node of the $5 \mathrm{G}$ wireless access network, its features of distribution, virtualization, and software and hardware decouping make it one of the effective entrances to the boundary [14]. Using software-defined networking / virtualization / cloud computing and other technologies, the flexible deployment adapts to different application scenarios. And it provides low-latency and highly reliable personalized network services for enterprises and vertical industries [15].

In terms of wireless frequency band resources, $230 \mathrm{MHz}$ is the electricity wireless private network frequency band [16], and there are ready-made core network resources in the experimental units, and its maturity remains to be observed. Its single station covers a wide area and has good penetration. When the antenna is about $50 \mathrm{~m}$ high, the base station generally covers about 2 kilometers in the urban area and $4-5$ kilometers in the suburbs; $1800 \mathrm{MHz}$ is used by subway / rail transit / civil aviation in the $4 \mathrm{G}$ wireless band, its terminal module technology is more mature than $230 \mathrm{MHz}$, and its application terminals are more diverse. When the antenna height is about $20-50 \mathrm{~m}$, the base station generally covers about 1 kilometers in urban areas, and the suburbs can cover 2-3 kilometers, and the penetration is average.

\subsection{Micro Integrated Communication Base Station System}

The micro integrated communication base station system is an integrated and fast integrated communication device that can complete the tower installation of the base station antenna feed platform, including the pole body, integrated equipment compartment, power supply system, and selfstabilizing foundation. The upper part of the pole body is installed with the antenna feed system and lighting monitoring equipment.

The miniature integrated microwave tower is set at a height of about $20-30 \mathrm{~m}$. A portable telescopic antenna can be added to the emergency communication vehicle or a small field base station in the field. The height of the portable telescopic antenna is $15-25$ meters. The single hop relay distance is within 5 kilometers and 5 jumps around [17].

\section{Solutions and Main Functional Modules}

Based on the investigation of the above key technologies, an emergency communication network architecture based on large-capacity digital microwave technology is proposed. Temporary LTE small base stations (operating frequency band is $230 \mathrm{MHz}$ or $1800 \mathrm{MHz}$ ) and RF-Mesh wireless ad hoc network equipment are deployed in the emergency area. Base stations and ad hoc network devices surround supplementary multimedia stream acquisition equipment such as drones and individual terminals. And then complete the fiber-optic network between base stations via large-capacity digital microwave stations, and use microwave jumps to complete interoperability with provincial company emergency command centers through the power backbone transmission network or satellite communication system.

\subsection{Solution}

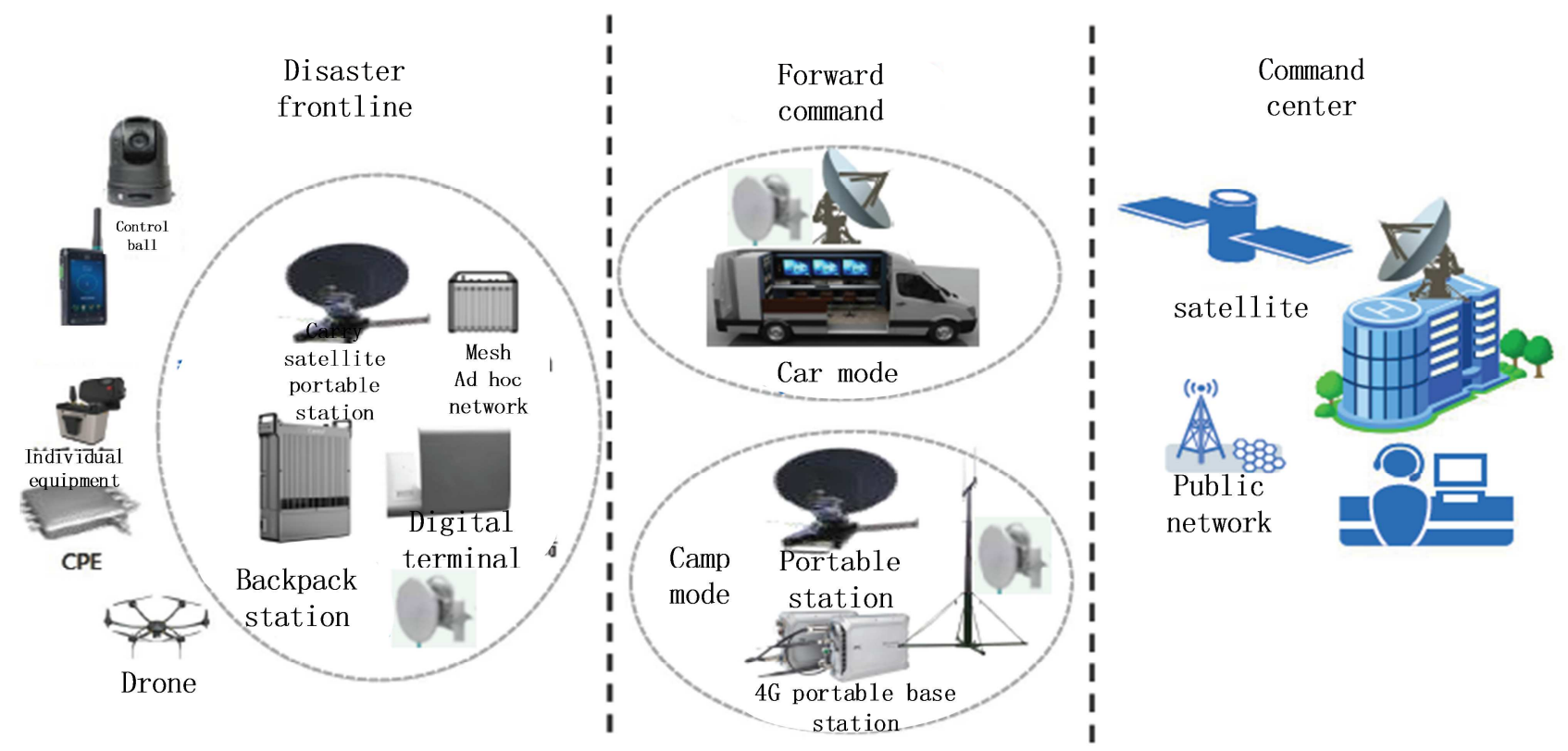

Figure 3. Heterogeneous networking architecture.

The specific network structure is shown in Figure 3. This network architecture can quickly set up an emergency support communication system when the emergency area is powered off and the operator's network is paralyzed. On the 
front line of disasters, LTE-230 or LTE-1800 4G piggybacked base stations are used to solve the problem of local communication coverage, and RF-Mesh wireless ad hoc network equipment is used to solve the problem of communication access coverage in the field or buildings with poor LTE signal coverage. Deploy emergency vehicle equipment at the forward command or set up a camp convergence node to receive multimedia signal backhaul from the disaster front line. The emergency base station kit for the camp mode is a small container with microwave terminal transmission equipment and all application equipment built in, eg. emergency terminal nodes such as cameras, man-machine, switch, and LTE emergency base station. So that we can use microwave to jump to the local county power supply company's power optical transmission overlay network to communicate with the emergency subcenter. Satellite communication or backbone transmission networks can also be used to establish communication channels between emergency areas and national, provincial or command centers.

The main advantages of the LTE-230 base station are the long signal coverage between the base station and the terminal, and the strong penetration. The main quality of the LTE1800 is its large bandwidth capacity, diverse product system and high technology maturity. The advantage of RF-Mesh wireless ad hoc network technology is that the network is flexible and convenient, and the response speed is fast. It can be used in the form of a backpack-type soldier. Rescuers can carry it to enter rescue areas that cannot be covered by LTE signals or have poor coverage. Terminal node signals are transmitted through multiple Mesh nodes to the ad hoc network node closest to the field LTE small base station or emergency command vehicle, and then through the network cable send the signal to the command center through the channel of the LTE base station or emergency command vehicle. The distance between RFMesh wireless ad hoc network equipment nodes is within $2 \mathrm{KM}$ (if it needs to penetrate walls or buildings, the effective distance of the signal will be reduced accordingly). In theory, the transmission hops of the ad hoc network only receive the transmit and power limits. In the case of 5-hop signal attenuation, it can provide a $2-4 \mathrm{M}$ bandwidth data channel [18].

\subsection{Small Base Station System}

It can be seen from Figure 4 that the portable base station has the characteristics of small size, mobile portability, rich functions, high integration and strong reliability. Quick startup, one-click start-up, emergency command network set up within 10 minutes; high integration, integrated PDT. [18] Trunk-like services, core network, baseband and RF unit; easy to carry, small size, light weight and mobile rod; There are various backhaul methods, including optical transmission, Ethernet, microwave, and RF-Mesh ad hoc network systems. Rich functions support capabilities, supporting a full range of PDT services, video surveillance, Ethernet data and other services; Strong environmental adaptability, waterproof and dustproof [19], high anti-vibration performance, wide temperature adaptability range. There are many application scenarios; energy-saving and power-saving, unified power supply interface, lower power consumption, energy-saving and power-saving, and low power consumption of the whole machine.

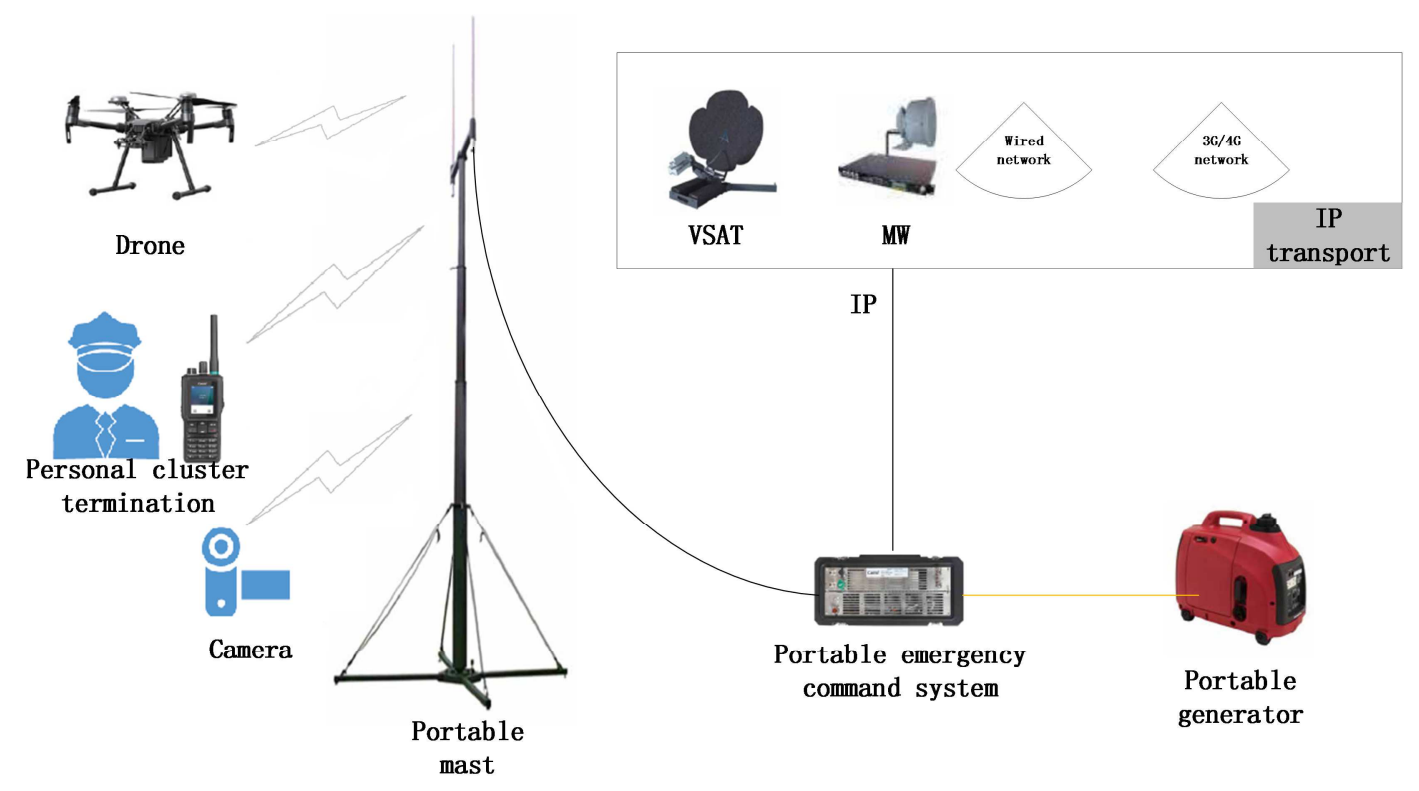

Figure 4. Portable base station platform equipment schematic.

\subsection{Vehicle Satellite Command System}

The satellite communication system provides a stable and reliable transmission link for the emergency command vehicle and the fixed command center. And it realizes the transmission of voice, data and video, and ensures the reliable operation of communication, command and dispatch. 
In the emergency command system, the satellite communication system includes a ground satellite master station and a satellite vehicle station. The ground satellite master station is generally located on the ground command center and provides a connection between the command vehicle and the command center. The satellite vehicle station uses a static satellite antenna, and an automatic antenna control system is used to achieve fast satellite alignment [20]. Through the integration of satellite communication systems, the communication link between the emergency forward command and the rear command center is established to ensure command and dispatch in emergency scenarios.

\subsection{Backpack Emergency Command System}

It can be seen from Figure 5, the backpack emergency command system is highly integrated with baseband, radio frequency, core network, and multimedia dispatching, and can quickly complete the deployment of on-site LTE emergency communication networks. It can provide LTE-based professional cluster calls, multimedia scheduling, real-time video backhaul, Geographic Information System (GIS) positioning, and full-duplex audio and video calls. The system supports multiple backhaul methods, including satellite, mesh ad hoc network, optical fiber, microwave, and public network.

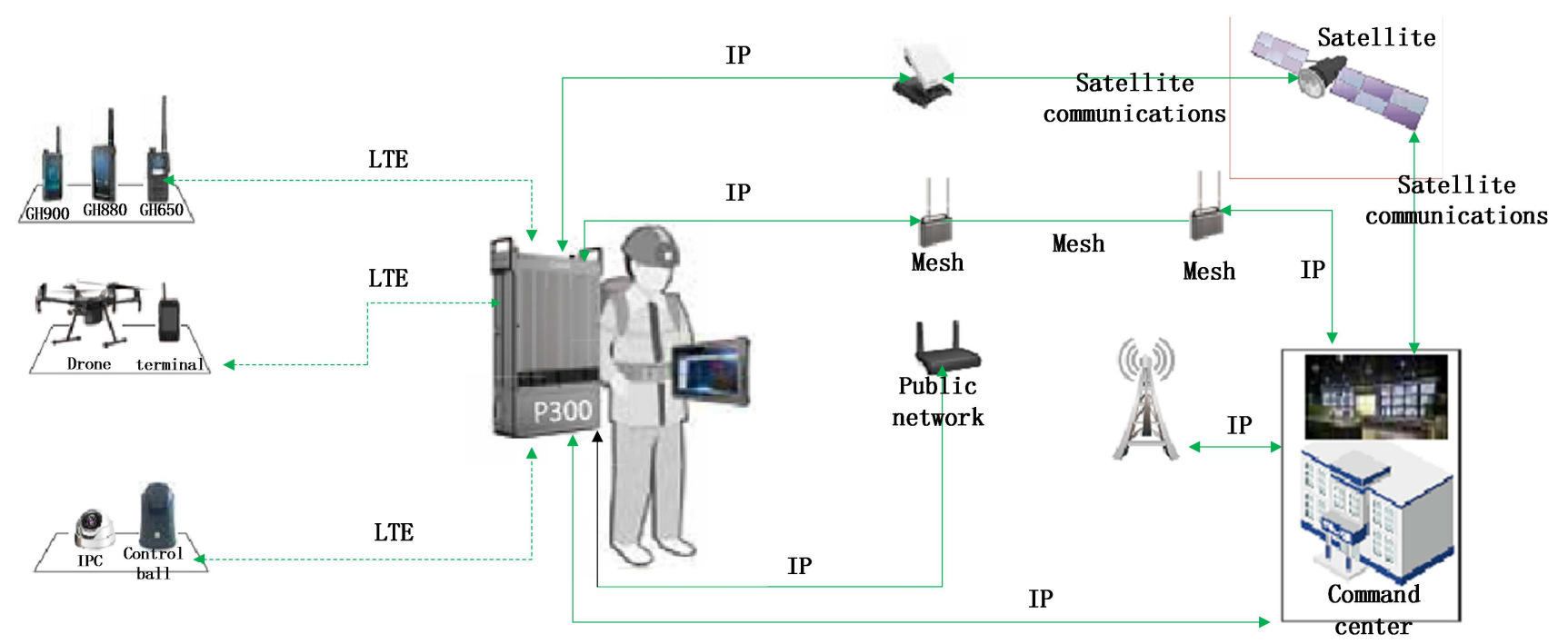

Figure 5. Collision emergency command system communication interconnection structure.

\subsection{UAV high Altitude Base Station System}

As shown in Figure 6, the LTE base station /Mesh base station can be hoisted to the sky by UAV to realize audio and video cluster communication over a wider area, which can quickly recover the long-distance communication signal in case of largescale communication interruption caused by natural disasters such as earthquake, geology and flood.

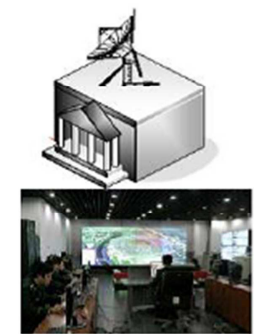

Rear command center
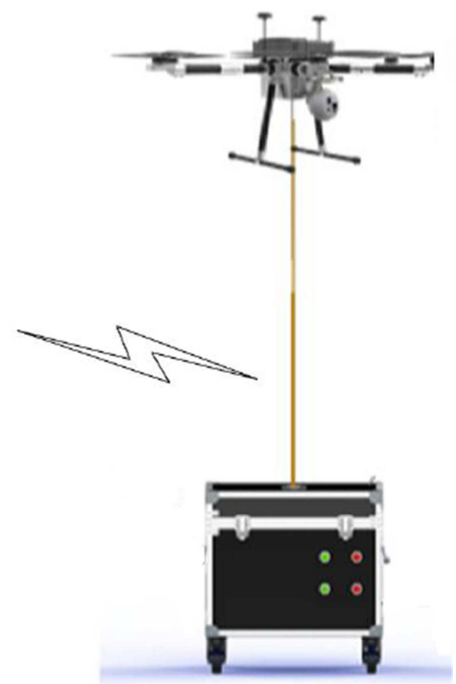

Drone control

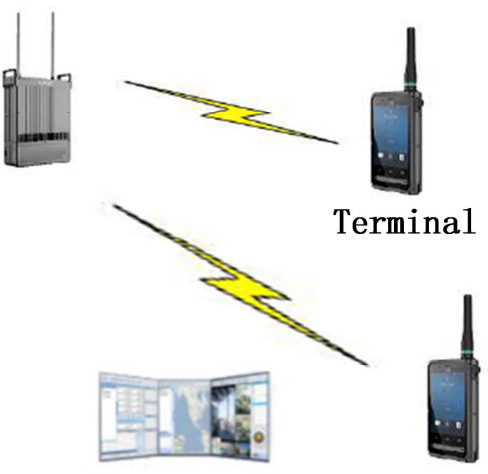

Termina1

Figure 6. High altitude base station schematic. 


\section{Application Scenario Display}

\subsection{Natural Disaster Emergency Wireless Communication Guarantee}

The solution in Figure 7 adopts the networking mode of converged terminal + satellite phone + LTE piggyback base station + Mesh base station. The Mesh base station is deployed in the forward command to realize the connection between the forward command and front-line rescue; The first-line rescue LTE piggyback station can be placed at a high place to provide first-line wide-range wireless signal coverage. At the same time, the Mesh base station can also be used as an extension of first-line rescue signal coverage to provide efficient and reliable protection for natural disaster emergency wireless communications.
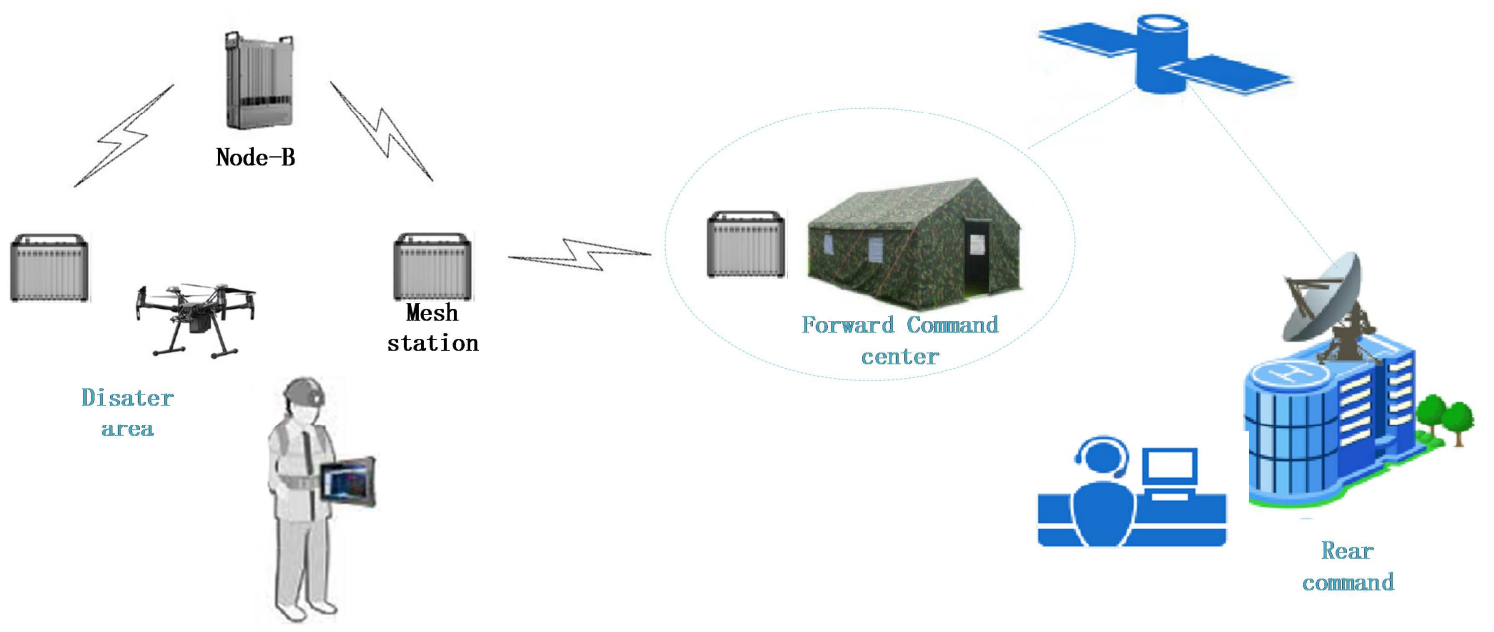

Figure 7. Disaster relief scenario networking schematic.

\subsection{Emergency Wireless Communication Guarantee for Transmission Lines}

The solution in Figure 8 adopts a networking method of a converged terminal + a Tiantong phone + an LTE-backed base station + a Mesh ad hoc network + a satellite portable station + a large-capacity digital microwave. Deploying a LTE portable base station at the front command to achieve wireless coverage of the front command; the satellite is used to realize the communication between the front and the squadron to ensure the connection between the squadron and the forward command. The mesh ad hoc network is used in combination with the LTE piggyback station to ensure the forward communication of the squadron. This way facilitates the real-time two-way audio and video communication between the squadron and the forward command.

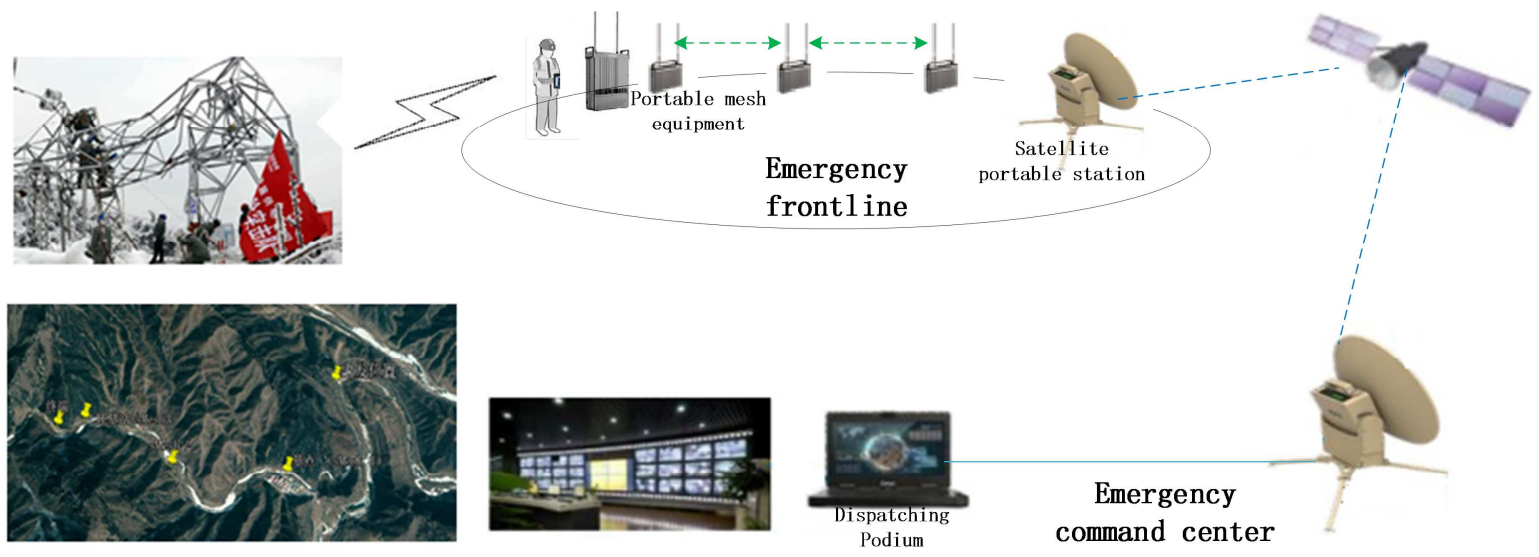

Monitor screen

Figure 8. Power line scene networking.

\section{Conclusion And Perspectives}

It is foreseeable that in the future, electricity emergency communications will present a trend of multiple-situation awareness. The networking scheme proposed in this paper has greatly improved the efficiency of setting up an on-site emergency system. Under extreme disaster scenarios, a variety sensing technologies make use of the unique advantages of their respective technologies, which can 
achieve multi-level command at the provincial, city and county levels of the company. At the same time, it can achieve wide coverage while ensuring the key target areas, which has certain promotion and application value.

\section{Acknowledgements}

This work has been funded by the Zhejiang Electric Power Company. Thanks for their efforts and fund that leads to complete this research and development.

\section{References}

[1] WANG Haitao. Analysis of the development status and technical means of emergency communication. China Radio, 2010 (11): 49-51

[2] JIA Haijun, ZHANG Yue. Application of Conference Call Technology in the Development of Public Security Emergency Command and Dispatching System. Computer Engineering \& Software, 2014 (4): 127-129.

[3] JIANG Weiyong, WU Wenchuan, ZHANG Boming, etc. Net-work Model Reconstruction in Online Security Early Warning System. Automation of Electric Power Systems, 2007, 31 (21): 5-9.

[4] FAN Kai. Research on a new generation of emergency command and dispatch communication system. Communication technology, 2011, 44 (3): 35-37.

[5] ZHANG Weiguang, YU Gang, LI Hongliang. Rapid deployment plan for mobile mobile communication sys-tems in emergency operations. Telecommunication Engi-neering, 2010, 50 (4): 11-14.

[6] Ramrekha T A, Politis C. An adaptive qos routing solution for manet based multimedia communications in emergency cases, International Conference on Mobile Lightweight Wire-less Systems. Berlin, Gem Heidelberg, Berlin: Springer, 2009: 7484.

[7] Ellis A D, Tan M, Iqbal M A, et al. $4 \mathrm{~Tb} / \mathrm{s}$ transmission reach enhancement using $10 \times 400 \mathrm{~Gb} / \mathrm{s}$ super-channels and polariza-tion insensitive dual band optical phase conjugation. Journal of lightwave technology, 2016, 34 (8): 1717-1723.

[8] Lichtensteiger B, Bjelajac B, Müller C, et al. RF mesh systems for smart metering: System architecture and performance, 2010 First IEEE International Conference on Smart Grid Communications. Washington, USA: IEEE, 2010: 379384.
[9] HONG Xijun, CHE Kenan, ZHANG Ji. Research on Wireless Ad Hoc Network Routing Protocol. Computer Engineer-ing (8): $110-112$.

[10] ZHOU Chao, ZHANG Xinggong, GUO Zongming. Multiuser video transmission optimization method for MIMO multi-hop wireless network. Journal of Software, 2013, 24 (2): 279-294.

[11] YU Hai, CAO Lei. WiFi-based wireless mesh (Mesh) network-ing technology. modern electronics technique, 2011, 34 (10): 120-122.

[12] MA Yufeng, GONG Shenguang, HU Xiulin, etc. Cellular wire-less communication network call admission control analysis. Journal on Communications, 2006, 27 (5): 108-114.

[13] SHU Peilian, LI Yi, JIANG Zhaojin. Comparative Analysis of Several Small Mobile Communication Base Stations. Mobile communications, 2015 (9): 82-86.

[14] Lutsiak M E C, Semnani R T, De Pascalis R, et al. Inhibition of $\mathrm{CD} 4+25+\mathrm{T}$ regulatory cell function implicated in enhanced immune response by low-dose cyclophosphamide. Blood, 2005, 105 (7): 2862-2868.

[15] Guttman E. Service location protocol: Automatic discovery of IP network services. IEEE Internet Computing, 1999, 3 (4): 71-80.

[16] LI Wenwei, CHEN Baoren, WU Qian, etc. Application Research of TD-LTE Power Wireless Broadband Private Network Technology. Telecommunications for Electric Power System, 2013, 33 (11): 82-87.

[17] ZHANG Shuling, JIANG Xinhua, Zou Fumin. Research and op-timization of multi-hop Mesh network performance based on open source code. Science technology and engineering, 2007, 7 (22): 5950-5953.

[18] LI Wanchen, CHEN Yuxian, ZHANG Jin, etc. Multi-channel multimedia data transmission system based on $3 \mathrm{G}$ wireless net-work [J]. Journal of Harbin University of Commerce (Natural Science Edition), 2013 (1): 79-81.

[19] WANG Wenxi, WANG Binheng, PU Jifeng. Structural design of IP65 small explosion-proof motor. explosion proof machine, 1999 (02): 22-24.

[20] WU Wenping, WANG Xiaobing. Design and Implementation of Fast Star Device Based on ARM9. modern electronic technology, 2009, 32 (19): 53-55. 